\title{
Papillary renal cell carcinoma embedded in an oncocytoma: Case report of a rare combined tumour of the kidney
}

\author{
István Sejben, MD; Zoltán Szabó, PhD, MD; Nándor Lukács, MD; ${ }^{+}$Márta Loránd, MD; Farkas Sükösd, MD;* \\ Gábor Cserni, $P h D, M D, D S c^{*}$
}

*Department of Pathology, Bács-Kiskun County Teaching Hospital, Kecskemét, Hungary; †'Department of Urology, Bács-Kiskun County Teaching Hospital, Kecskemét, Hungary; §Department of Radiology, Bács-Kiskun County Teaching Hospital, Kecskemét, Hungary; ${ }^{*}$ Department of Pathology, University of Szeged, Szeged, Hungary

Cite as: Can Urol Assoc J 2013;7(7-8):e513-6. http://dx.doi.org/10.5489/cuaj.414 Published online on July 2, 2013.

\section{Abstract}

An asymptomatic 1-cm large papillary renal cell carcinoma (RCC) embedded in a 3.5-cm large oncocytoma was diagnosed and removed by right nephrectomy in a 68-year-old male investigated for the abdominal symptoms associated with cholelithiasis. The papillary RCC displayed positive immunohistochemical stainings with cytokeratin 7, alpha-methylacyl-CoA racemase and vimentin and was negative for the E-cadherin and CD117 immunostains, whereas the oncocytoma part showed opposite staining patterns. No gains of chromosomes 7 and 17 or loss of chromosome $Y$ was detected in the papillary carcinoma by fluorescent in situ hybridization with centromeric enumeration probes. This finding is in keeping with the morphologic diagnosis of type 2 papillary RCC reported to have lower rates of these characteristic chromosomal changes. The combination of papillary RCC and oncocytoma, two tumours of different postulated origin, is extremely rare. It may represent a simple coincidence, but 2 previous cases and our current one share a few features, including the intimate embedment of the papillary RCC in the oncocytoma, the small size of the RCC and the old age of the patients. This case raises the point that renal oncocytomas can contain a hidden malignant tumour.

\section{Introduction}

Renal cell neoplasms are supposed to derive from or show differentiation toward different parts of the renal epithelium, as highlighted by immunohistochemical staining patterns and differential expression of some marker proteins. ${ }^{1-3}$ There are reports on renal tumours with hybrid features between chromophobe renal cell carcinoma (RCC) and oncocytoma, both thought to arise from the distal tubular epithelium. ${ }^{4}$ Sometimes RCC arises within an oncocytoma. ${ }^{5}$ However, the combination of oncocytoma and papillary RCC, 2 renal neoplasms of different origin, is very rare. ${ }^{6,7}$ We present a case of an unusual combination of these 2 tumours, a papillary RCC buried in an oncocytoma.

\section{Case report}

A 68-year-old male with long-standing hypertension was admitted to our hospital because of right upper abdominal pain. Abdominal ultrasound and computerized tomography (CT) revealed a thick-walled gallbladder with common bile duct stones and a 4-cm mass in the upper pole of the right kidney (Fig. 1). The clinical diagnosis of choledocholithiasis associated with acute cholecystitis and a renal tumour were established. After endoscopic sphincterotomy, the symptoms related to the common bile duct stone vanished and the patient underwent right nephrectomy.

The nephrectomy specimen was fixed in $10 \%$ neutral buffered formalin for 36 hours. We embedded 3-mm-thick representative tissue sections in paraffin wax and sections of 4 to $5 \mu \mathrm{m}$ were cut and stained with hematoxylin and eosin (H\&E) for light microscopy. The primary antibodies used for immunohistochemistry are shown in Table 1.

\section{Results}

The parenchymal tumour in the nephrectomy specimen was circumscribed, homogeneous, yellowish-brown and measured $35 \times 35 \times 30 \mathrm{~mm}$. Microscopically, all but one slide showed polygonal cells forming nests and tubules that had abundant eosinophilic, granular cytoplasm and uniform, round central nuclei. Neither mitotic figures nor clear cell areas were detected. The tumour morphology was in keeping with the diagnosis of an oncocytoma. A papillary lesion with papillary and tubulopapillary structures measuring $1 \mathrm{~cm}$ in diameter was identified microscopically within this tumour. Eosinophilic, granular, but less voluminous cytoplasm and focal nuclear pseudostratification characterized this area. Cytoplasmic clearing was identified focally. Foamy macrophages and rare psammoma bodies occurred in the core of the papillae. The nuclei were generally of low grade, but areas with larger nuclear to cytoplasmic ratio and nucleoli visible at medium power magnification were also present resulting in a Fuhrman grade 2 overall (Fig. 2). The papillary carcinoma was present in a single block only, and attempts 


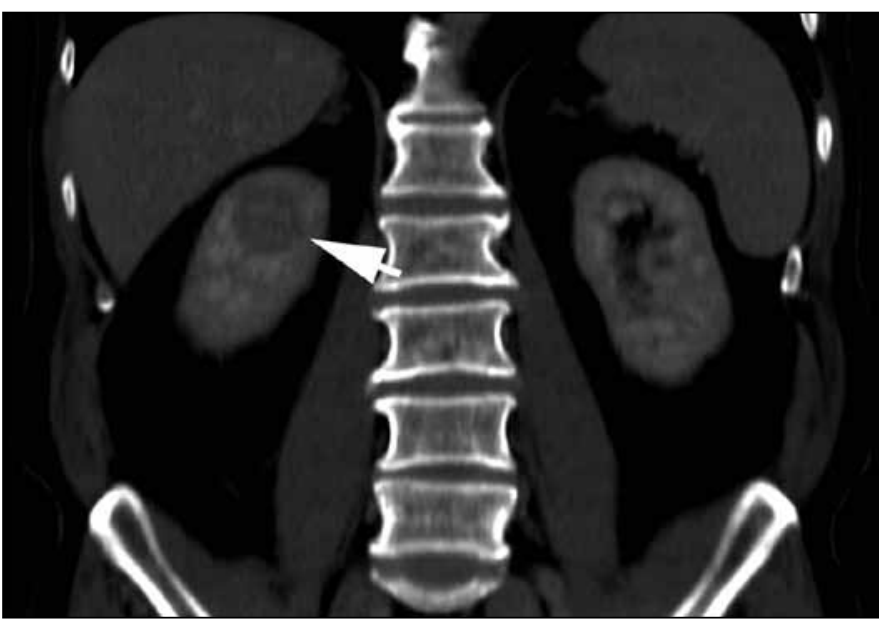

Fig. 1. Localization of the tumour on computed tomography scan. The arrow shows the circumscribed tumour in the upper part of the right kidney.

to identify any remnants of it grossly and in further tumour blocks taken failed.

The oncocytoma part showed diffuse positivity for E-cadherin and weak positivity for CD117, whereas it was negative for vimentin and alpha-methylacyl-CoA racemase (AMACR). It showed very focal cytokeratin 7 (CK7) positivity limited to small areas with more pleomorphic nuclei, which is a recognized feature of some oncocytomas. The papillary area was positive for CK7, AMACR and vimentin, but negative for CD117 and E-cadherin (Fig. 3). Neither parts were

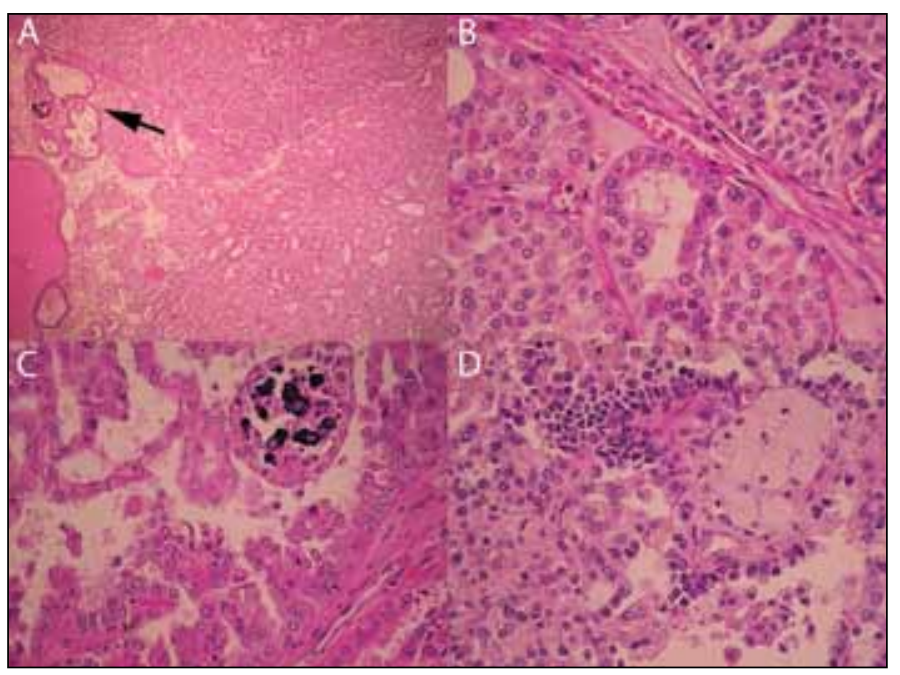

Fig. 2. Hematoxylin and eosin appearance of the composite tumour. A: Papillary renal cell carcinoma (RCC) (upper triangular area) separated from the oncocytoma by a thin pseudocapsule but also showing an irregular extension into it, with psammomatous calcification (arrow) $(\times 100)$. B: Part of the pseudocapsule separating the 2 tumour components. Note the cytoplasmic eosinophilia in both components: the low grade nuclei (in this field) and the higher nuclear to cytoplasmic ratio in the papillary RCC component (upper part) in comparison with the oncocytoma (lower part) $(\times 400)$. C: Psammomatous calcification, some nuclear pseudostratification and higher grade nuclei in this area of the papillary RCC $(\times 400)$. D: Foamy macrophages in a papillary core in the RCC $(\times 400)$.
Table 1. Primary antibodies used

\begin{tabular}{lccc}
\hline & Manufacturer & Clone number & Dilution \\
\hline CD10 & Cell Marque & $56 \mathrm{C} 6$ & $1: 50$ \\
CK7 & Dako & OV-TL 12/30 & RTU \\
AMACR & Dako & $13 \mathrm{H} 4$ & RTU \\
CD117 & Dako & polyclonal & $1: 80$ \\
E-cadherin & Novocastra & 36B5 & $1: 40$ \\
vimentin & Dako & VIM 3B4 & RTU \\
synaptophysin & Cell Marque & polyclonal & RTU \\
chromogranin A & Cell Marque & LK2H10 & RTU \\
\hline RTU: ready to use. & & &
\end{tabular}

stained with CD10, chromogranin A and synaptophysin. Considering the size, the structure of the lesions and their immunohistochemical staining patterns, the diagnosis of type 2 papillary RCC in an oncocytoma was made.

A fluorescent in situ hybridization (FISH) assay was used to assess the numbers of chromosomes 7,17 and $\mathrm{Y}$, according to the manufacturer's instructions (Cytocell Ltd, Cambridge, UK). The chromosome enumeration probes hybridizing to the centromere of the relevant chromosomes (LPE007R, Alpha satellite 7 Red; LPE017R, Alpha satellite 17 Red; LPEOYCR, Alpha satellite $Y$ Red) were labelled with spectrum red. The results were evaluated with a ZEISS Axio Imager Z2 microscope. Chromosome copy numbers were counted in 20 cells for each assay, and values of signal/cell ratio greater than 2.8 were defined as trisomy. The FISH assays demonstrated no gains of chromosomes 7 or 17, and no loss of chromosome Y. The patient did not receive adjuvant therapy. The followup was short, but he is alive without any signs of recurrence or metastasis 8 months after nephrectomy.

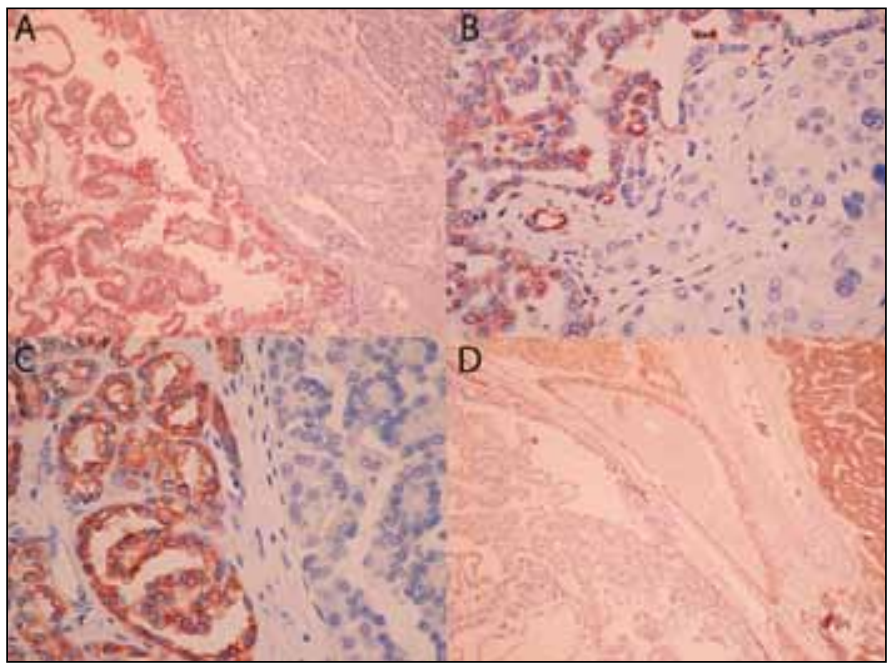

Fig. 3. Diagnostic immunohistochemistry of the 2 tumours components; AMACR (A), vimentin (B) and CK7 (C) positivity in the papillary RCC (left) in opposition with the negativity for these stains in the oncocytoma (right). E-cadherin displayed an opposite staining pattern (D). (A and D: $\times 100$; B and C: $\times 400$ ) 


\section{Discussion}

In the 2004 WHO classification of kidney tumours, oncocytoma is considered a benign neoplasm. It is characterized by eosinophilic granular large cells forming diffuse sheets and tubules in an oedematous stroma without papillary or clear cell areas. ${ }^{8}$ Immunohistochemically, oncocytomas exhibit positivity for E-cadherin and CD117, and negativity or focal positivity for CK7, AMACR and CD10. ${ }^{3,9}$ It is also recognized that oncocytomas can occur in Birt-Hogg-Dube syndrome, a rare autosomal dominant condition. ${ }^{10}$

Papillary RCC accounts for $10 \%$ of RCCs. Although size criteria of diagnosis always require caution, they are stated to be larger than $5 \mathrm{~mm}$ as opposed to papillary adenoma. ${ }^{8}$ Two subtypes are recognized. Type 1 tumours have papillae covered by a single layer of small uniform cells. In type 2 tumours, the cells covering the papillae are pseudostratified with eosinophilic cytoplasm and are usually of higher nuclear grade than type 1 papillary carcinomas. Immunohistochemically, papillary RCCs usually show positivity for CK7, AMACR, CD10 and negativity for CD117, E-cadherin. ${ }^{3,9,11}$

Although oncocytoma and RCC are separate entities, they can coexist in the same or the contralateral kidney. Chromophobe RCC and oncocytoma are suspected to be closely related and are thought to show a similar distal tubular phenotype, ${ }^{1-3}$ therefore their coexistence is not very surprising. However, oncocytomas and papillary RCCs originate from different cells, and the presence of a papillary RCC within an oncocytoma is extremely rare. We were only able to identify 2 reports of this coexistence. ${ }^{6,7}$ The first of a 75-year-old male whose type I papillary RCC of $7 \mathrm{~mm}$ was embedded in an oncocytoma measuring $1.5 \mathrm{~cm}$ in diameter. The papillary tumour in this case was easily identifiable, as it was clearly delineated and obviously different from the surrounding oncocytoma on H\&E stained sections. Unlike in our case, the cells of the papillary neoplasm displayed trisomy of chromosome $7 .{ }^{6}$ The second case occurred in a 73-year-old female and consisted of a $1.5-\mathrm{mm}$ diameter type II papillary RCC in a 36-mm diameter oncocytoma. No chromosomal copy numbers were analyzed, but the typical morphology and immunohistochemistry confirmed the diagnosis. ${ }^{7}$ Both cases were treated with partial nephrectomy.

Our diagnosis of a combined renal tumour consisting of a papillary RCC in an oncocytoma was established on microscopic morphological features and immunohistochemistry. In some areas, the tumours were clearly distinct from each other, but at other foci the 2 tumours merged and showed a diagnostically challenging morphology. Immunostains were of great help in distinguishing the 2 components. Gains of chromosomes 7, 17 and loss of chromosome $Y$ have been described as characteristic of papillary renal neoplasms, ${ }^{12}$ and only very rare tumours were reported to lack these marker changes. ${ }^{13}$ The centromeric probes identified none of the characteristic chromosomal changes, in keeping with their lower incidence in type 2 papillary RCC. ${ }^{14}$

According to the current trends in urology, small renal masses $(\leq 4 \mathrm{~cm})$ are often treated by ablative therapies or active surveillance. Prior to such therapeutic approaches, renal biopsy should be performed to establish a histological diagnosis. ${ }^{15}$ Image-guided percutaneous core needle biopsy of renal masses has more than $90 \%$ sensitivity for the detection of malignancy. ${ }^{16}$ However, combined/hybrid tumours consisting of benign and malignant areas can be misdiagnosed, because the biopsy may miss either tumour component or the other. Oncocytomas may sometimes harbour a malignant tumour, especially chromophobe RCC, or as in our case another malignancy. ${ }^{17}$ This should be considered when biopsy is used to direct management.

\section{Conclusion}

Our literature review identified that papillary RCCs arising in, or embedded in, an oncocytoma are definitely rare. Although the intimate relationship of the 2 tumors would suggest some causal relationship, the rarity of this combination, the different postulated origins of the 2 components would make it more likely to represent coincidence. Of the 3 papillary RCCs, 2 were type 2 . All were minute pT1a carcinomas occurring in elderly patients over 65 , and although no information on follow-up was published in previous reports, ${ }^{6,7}$ the reported prognostic features were rather favourable. Partial nephrectomy was the surgical treatment for the previously reported cases, but nephron-sparing surgery was not considered in our current case because the 4-cm tumour was close to the renal hilum and the diagnosis of oncocytoma was not considered preoperatively. Biopsies from combined tumours like this one can pose diagnostic challenges to urologists and pathologists.

Competing interests: None declared.

This paper has been peer-reviewed.

\section{References}

1. Shen SS, Krishna B, Chirala R, et al. Kidney-spectific cadherin, a specific marker for the distal portion of the nephron and related renal neoplasms. Mod Pathol 2005; 18:933-40. http://dx.doi.org/10.1038/modpathol.3800373

2. Skinnider BF, Folpe AL, Hennigar RA, et al. Distribution of cytokeratins and vimentin in adult renal neoplasms and normal renal tissue: potential utility of a cytokeratin antibody panel in the differential diagnosis of renal tumors. Am J Surg Pathol 2005;29:747-54. http://dx.doi.org/10.1097/01.pas.0000163362.78475.63

3. Al-Ghawi $\mathrm{H}$, Asoyo OA, Truong $\mathrm{LD}$, et al. Application of immunohistochemistry to the diagnosis of kidney tumors. Pathol Case Rev 2010;15:25-34. http://dx.doi.org/10.1097/PCR.0b013e3181d51c70

4. Petersson F, Gatalica Z, Grossmann P, et al. Sporadic hybrid oncocytic/chromophobe tumor of the kidney: a clinicopathologic, histomorphologic, immunohistochemical, ultrastuctural, and molecular cytogenetic study of 14 cases. Virchows Arch 2010;456:355-65. http://dx.doi.org/10.1007/s00428-010-0898-4 
Sejben et al.

5. Davis $\mathrm{CJ} \mathrm{Jr}$, Sesterhenn IA, Mostofi FK, et al. Renal oncocytoma. Clinicopathological study of 166 patients. J Urogen Pathol 1991;1:41-52.

6. Rowsell C, Fleshner N, Marrano P, et al. Papillary renal cell carcinoma within a renal oncocytoma: case report of an incidental finding of a tumour within a tumour. J Clin Pathol 2007;60:426-8. http://dx.doi.org/10.1136/icp.2006.041129

7. Floyd MS, Javed S, Pradeep KE, et al. Composite oncocytoma and papillary renal cell carcinoma of the kidney treated by partial nephrectomy: A case report. ScientificWorldJournal 2011;11:1173-7. http://dx.doi.org/10.1100/tsw.2011.120

8. Lopez-Beltran A, Scarpelli M, Montironi R, et al. 2004 WHO classification of the renal tumors of the adults. Eur Urol 2006;49:798-805. http://dx.doi.org/10.1016/i.eururo.2005.11.035

9. Truong LD, Shen SS. Immunohistochemical diagnosis of renal neoplasm. Arch Pathol Lab Med 2011;135:92-109.

10. Pavolich CP, Walther MM, Eyler RA, et al. Renal tumors in the Birt-Hogg-Dube syndrome. Am I Surg Pathol 2002;261:1542-52.

11. Delahunt B, Eble JN. Papillary renal cell carcinoma: a clinicopathologic and immunohistochemical study of 105 tumors. Mod Pathol 1997; 10:537-44.
12. Kovács $G$, Füzesi L, Emanuel $A$, et al. Cytogenetics of papillary renal cell tumors. Genes Chromosom. Cancer 1991;3:249-55. http://dx.doi.org/10.1002/gcc.2870030403

13. Bálint I, Szponar A, Jauch A, et al. Trisomy 7 and 17 mark papillary renal cell tumours irrespectively of variation of the phenotype. J Clin Pathol 2009;62 892-95. http://dx.doi.org/10.1136/icp.2009.066423

14. Kuroda $N$, Toi $M$, Hiroi $M$, et al. Review of papillary renal cell carcinoma with focus on clinical and pathobiological aspects. Histol Histopathol 2003; 18:487-94.

15. Jewett MAS, Finelli A, Kollmansberger $C$, et al. Management of kidney cancer: Canadian Kidney Cancer Forum Consensus update 2012. Can Urol Assoc J 2012:6:16-22.

16. Maturen KE, Nghiem HV, Caoili EM, et al. Renal mass core biopsy: Accuracy and impact on clinical management. Am J Roentgenol 2007:188:563-70. http://dx.doi.org/10.2214/AJR.06.0220

17. Schmidbaver J, Remzi M, Mamarsadeghi M, et al. Diagnostic accuracy of computed tomography-guided percutaneous biopsy of renal masses. Eur Urol 2007;53:1003-11. http://dx.doi.org/10.1016/.eururo.2007.11.041

Correspondence: Dr. István Sejben, Department of Pathology, Bács-Kiskun County Teaching Hospital, Nyiri ưt 38., H-6000 Kecskemét, Hungary; fax: +36 76 481219; sejbenisł@freemail.hu 